\title{
Images of the month 4: Cutaneous amyloidosis: a clinical challenge
}

\author{
Authors: Morteza Moatamedi ${ }^{A}$ and Mohammad H Derakhshan ${ }^{B}$
}

KEYWORDS: Amyloidosis, diagnosis, management

\section{Case presentation}

A 63-year-old woman presented with a 6-year history of discoloured areas on right palm, right middle and ring fingers. She was asymptomatic except for one episode of bleeding from the affected area following minor trauma. She was right handed.

Past medical history included premature labour followed by total abdominal hysterectomy, irritable bowel syndrome, essential hypertension, duodenal ulcer, cerebrovascular accident and depression. She was an ex-smoker. Her current medication included aspirin. There was no drug allergy.

Examination revealed dull red, flat, non-pulsatile areas on palm (Figs 1 and 2). There was no hyperkeratosis or ulceration. Small venous lake on right upper lip was noted.

\section{Investigations}

Blood tests including full blood count, biochemical profile, liver function test and C-reactive protein reported within normal values. Immunoelectrophoresis resulted in slight increase in immunoglobulin (Ig) M but no para-protein band. There was no evidence of Bence Jones proteinuria.

Skin biopsy was performed with following findings (Fig 3). Epidermis: thick keratin.

Dermis: oesinophilic material, probably fibrin with loose stroma and loose red cells, some ectasia of small blood vessels but no haemosiderin.

Histology sample re-examined. Patient declined to have rectal biopsy.

Diagnosis: cutaneous amyloidosis.

\section{Discussion}

Amyloidosis is defined by extra-cellular deposition of proteinaceous substance or various insoluble proteins that results in considerable tissue dysfunction. All types of amyloid consist of a

Authors: A locum consultant dermatologist, Salford Royal NHS Foundation Trust, Salford, UK; ${ }^{B}$ associate professor and clinical epidemiologist, University of Glasgow, Glasgow, UK

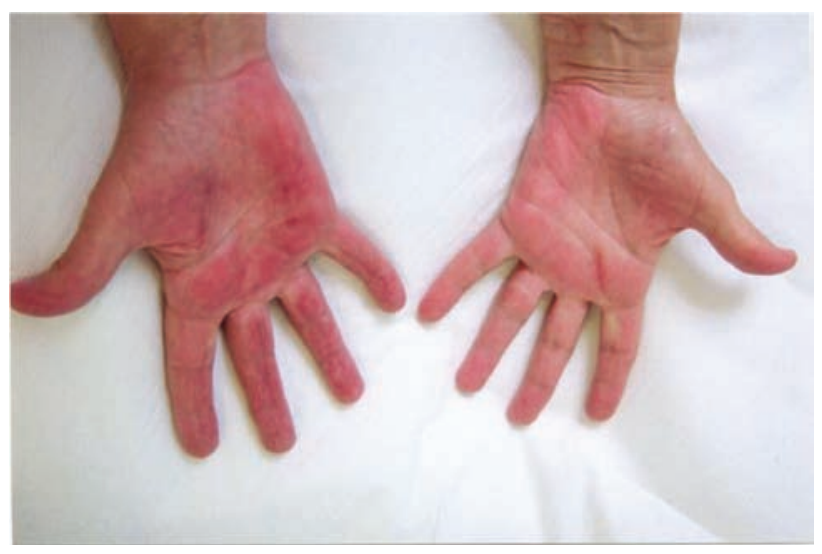

Fig 1. Clinical presentation - erythematous rash on palm of hands.

major fibrillar protein that defines the type of amyloid plus various minor components. It may affect heart, kidney, gastrointestinal tract, nervous system and skin (dermal layer). It was first reported by Rudolph Virchow, German physician in 1854. Amyloidosis is usually classified as primary or secondary according to the underlying cause. Recent classification is based on biochemical findings (Table 1).

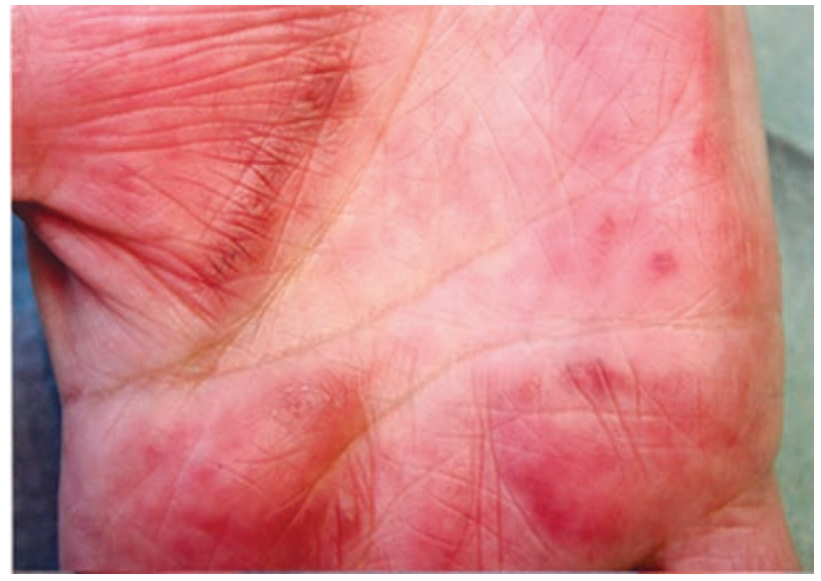

Fig 2. Clinical presentation - dark red discoloured thickened skin, no hyperkeratosis. 


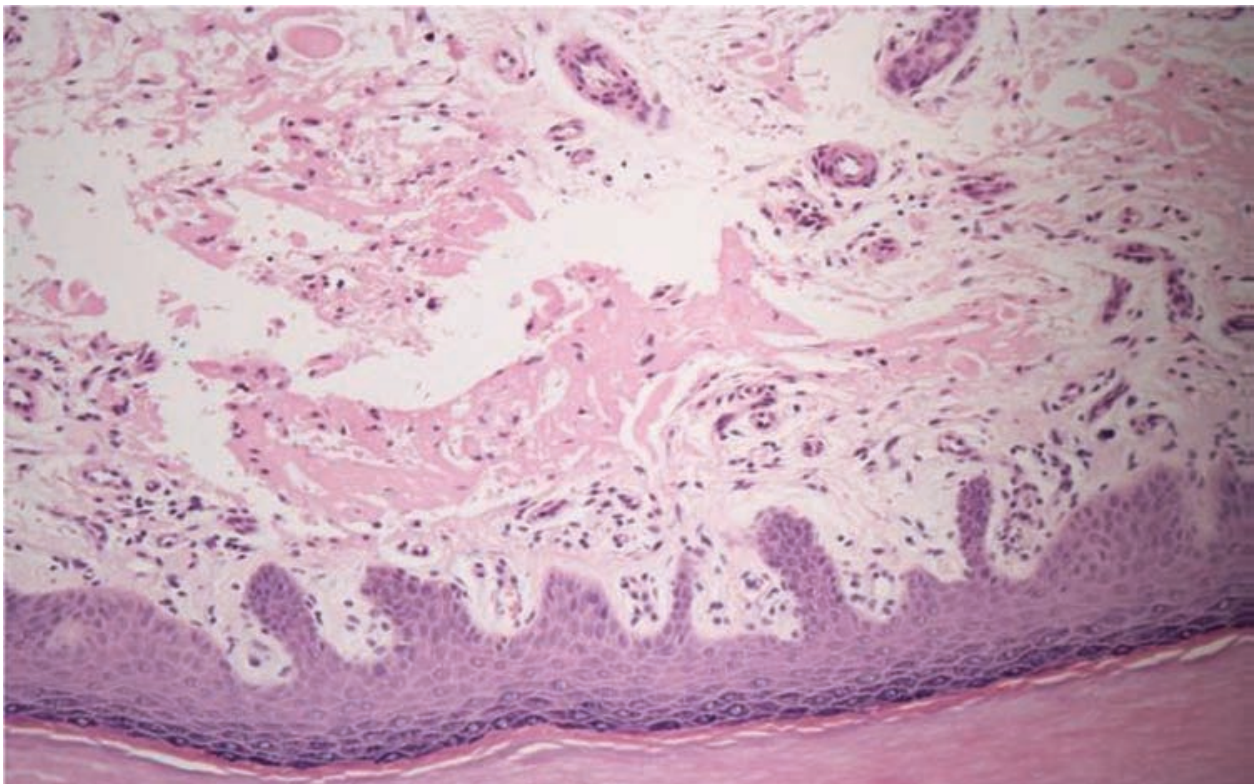

Fig 3. Histological findings haematoxylin and eosin stained slide, amyloid deposits as extracellular eosinophilic material.

In this case clinical findings suggested primary localised cutaneous amyloidosis (PLCA). In this condition, there is deposition of amyloid in previously normal skin with no evidence of deposits in internal organs. Amyloids build up in the skin, specifically in the 'dermal papillae' between the epidermis and the dermis. It is associated with chronic friction or epidermal damage - friction amyloidosis. According to the clinicopathological observations, PLCA is classified as macular, papular or lichen amyloidosis, nodular or tumefactive and familial (dyschromic).

Macular form is presented with flat dusky-brown or grey macules that may coalesce with variable degree of itchiness. It is usually symmetrical and affects upper back, chest and occasionally arms. It presents in early adult life with female to male predominance. Pathological finding includes amyloid in papillary dermis with no involvement of blood vessels or adnexal structures. There are small amorphous globules. Epidermis has normal thickness.

Papular or lichen amyloidosis is the most common form. It presents with intense itchiness and multiple scaly red or brown papules that may coalesce into plaques. It is more common in males between 50-60 years old. Pathological finding includes focal deposits, large enough to expand dermal papillae displaced rete ridges.

The epidermis has irregular acanthosis and hyperkeratosis.

This case suggests macular form of PLCA although it differs from some usual clinical and pathological presentations.

Primary cutaneous amyloidosis has been reported in different body areas including eyelid ${ }^{1}$ and the auricular concha. ${ }^{2}$

\section{Key points}

> Amyloidosis is defined by extra-cellular deposition of proteinaceous substance or various insoluble proteins that results in considerable tissue dysfunction.

> In primary localised cutaneous amyloidosis (PLCA), there is deposition of amyloid in previously normal skin with no evidence of deposits in internal organs.

$>$ PLCA is classified as macular, papular or lichen amyloidosis, nodular or tumefactive and familial (dyschromic).

$>$ Cutaneous amyloidosis may be associated with systemic amyloidosis.

> Management of cutaneous amyloidosis include topical and systemic treatment, cryotherapy and surgical measures.

Cutaneous amyloidosis may be associated with systemic amyloidosis. There are case reports of sarcoidosis and IgA nephropathy in association with extensive macular amyloidosis. At least a subset of PLCA patients, especially those with extensive involvement, may have associated autoimmune/ immune disorders raising the possibility of a common underlying immune-mediated mechanism. ${ }^{3}$

Familial PLCA shows autosomal dominant inheritance, but there is clinical and genetic heterogeneity and variable clinical penetrance. $^{4}$

\section{Table 1. Classification of amyloidosis}

\section{Clinical type of amyloidosis}

Primary (immunnocytodyscrasia)

Secondary (chronic active disease) eg rheumatoid arthritis, osteomyelitis

Haemodialysis associated

Heredofamilial (commonly affected kidney), rare

\section{Amyloid fibril protein Precursor substance}

$\mathrm{AL}$ (amyloid L chain)

Monoclonal immunoglobulin light chain

$\mathrm{AA}$ (amyloid $\mathrm{A}$ ) Serum amyloid A protein (SAA)

$\beta 2$ - macroglobulin 
Management of PLCA is challenging. In this case, patient was treated with potent topical steroid with no significant improvement. Other options include calcipotriol, phototherapy, dimethyl sulfoxide, etretinate, acitretin, cyclophosphamide, ciclosporin, cryotherapy, dermabrasion, $\mathrm{CO}_{2}$ laser and surgical measures.

\section{References}

1 Mun KS, Pailoor J, Reddy SC. Primary localised deep cutaneous amyloidosis of the eyelid. Malays ] Pathol 2005;27:113-5.

2 Shimauchi T, Shin JH, Tokura Y. Primary cutaneous amyloidosis of the auricular concha: case report and review of published work. J Dermatol 2006:33:128-31.
3 Dahdah M], Kurban M, Kibbi AG, Ghosn S. Primary localized cutaneous amyloidosis: a sign of immune dysregulation? Int J Dermatol 2009:48:419-21.

4 Sakuma TH, Hans-Filho G, Arita K et al. Familial primary localized cutaneous amyloidosis in Brazil. Arch Dermatol 2009;145:695-9.

Address for correspondence: Dr Morteza Moatamedi, Dermatology Department, Salford Royal NHS Foundation Trust, Stott Lane, Salford M6 8HD, UK.

Email: moatamedi@hotmail.com

\section{Looking for jobs in medicine?} Find your next hospital job

Find out more and subscribe to the monthly Medicine Jobs bulletin at www.rcplondon.ac.uk/jobsboard 